\title{
FRACTURE STRAIN OF LPCVD POLYSILICON
}

\author{
Y. C. Tai and R. S. Muller
}

\author{
Berkeley Sensor and Actuator Center \\ An NSF/Industry/University Cooperative Research Center \\ Department of Electrical Engineering and Computer Sciences \\ and the Electronics Research Laboratory \\ University of California, Berkeley CA 94720
}

\begin{abstract}
A new polysilicon bridge-slider structure (Fig. 1), in which one end of the bridge is fixed and the other is connected to a plate sliding in two flanged guideways, is designed and fabricated to study the strain at fracture of LPCVD polysilicon. In the experiments, a mechanical probe is used to push against the plate end, compressing and forcing the bridge to buckle until it breaks. The distance that the plate needs to be pushed to break the bridge is recorded. Nonlinear beam theory is then used to interpret the results of these axially-loaded-bridge experiments. The measured average fracture strain of as-deposited LPCVD polysilicon is $1.72 \%$. High-temperature annealing of the bridgesliders at $1000{ }^{\circ} \mathrm{C}$ for one hour decreases the average fracture strain to $0.93 \%$.
\end{abstract}

\section{INTRODUCTION}

Polysilicon has been demonstrated to have useful applications for sensors and actuators. Moreover, complex polysilicon micromechanisms have been shown to be feasible $[1,2]$ using pin joints, gears, springs, and cranks. As a result, new devices like micro-motors and micro optical shutters are becoming possible. Proper design of these structures is hindered, however, by the lack of precise knowledge of the mechanical properties of polysilicon. Of these parameters, strain at fracture is particularly important.

For single-crystal silicon, Eisner [3] reported a maximum fracture strain of $2.03 \%$ measured on whiskers about one micrometer in diameter under tension. Pearson, Read, and Feldmann [4] reported a maximum fracture strain of $2.6 \%$ for silicon whiskers both grown from vapor and cut from bulk silicon. Moreover, it is found that below $600{ }^{\circ} \mathrm{C}$ there is little or no plastic flow in silicon whiskers about $20 \mu \mathrm{m}$ in diameter before fracture.

It is expected that polysilicon will also behave linearelastically before fracture at room temperature because grain boundaries in polysilicon can greatly block dislocation motion [6] and make the polysilicon more like an ideal brittle material. Based on this assumption, Fan, Tai, and Muller [5] reported preliminary experiments which determined an experimental fracture strain of polysilicon to be $1.7 \%$ using a spiral-spring-restrained pin-joint structure. However, such a spring-restrained pin-joint structure is not optimal for the fracture experiment. Moreover, it is generally accepted that a statistical method should be used to study the fracture strength in brittle materials [6].

We report here a systematic method to study the fracture strain of polysilicon. We introduce a new, easily implemented method using a bridge-slider structure to avoid the difficulties of handling small samples as reported in the silicon whisker experiments [3,4]. The bridge-slider structure is specially designed and processed to improve experimental accuracy.

\section{BRIDGE-SLIDER STRUCTURE}

Figure 1 shows an SEM photograph of the newly designed bridge-slider structure. The right end of the free-standing bridge, shown in Fig. 1, is anchored to the silicon substrate, while the left end is connected to a sliding plate guided by two flanges. The outer edges of the slider flanges are sawtooth shaped with a tooth pitch of $4 \mu \mathrm{m}$ to provide scales for locating the end of the slider. This sawtooth feature greatly simplifies our experiments. Figure 2 shows a cross section of the slider to demonstrate its translational freedom of motion. Clearly, the slider, made of second-layer polysilicon, is fully separated from the restraining elements that are made of first-layer polysilicon. The flanges one of which is circled in Fig. 2 allow the slider only to slide in and out of the plane of Fig. 2.

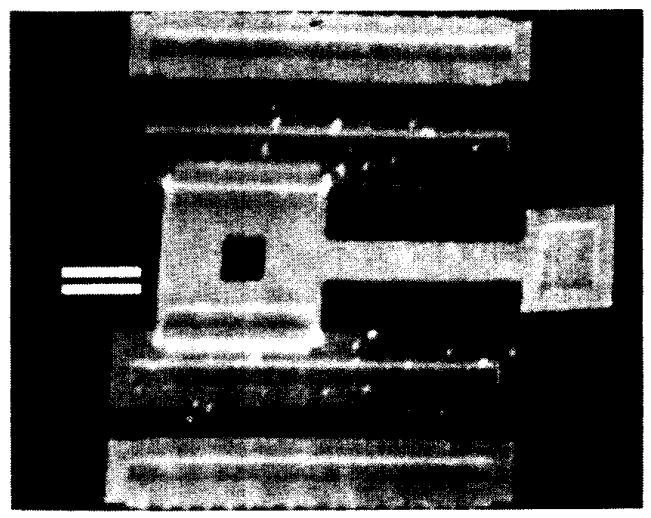

Figure 1 A bridge-slider structure. The bridge-slider is made of second-layer polysilicon. Integrated sawtooth scales are defined at the outer side of flanges. The marker is $30 \mu \mathrm{m}$ in length. 


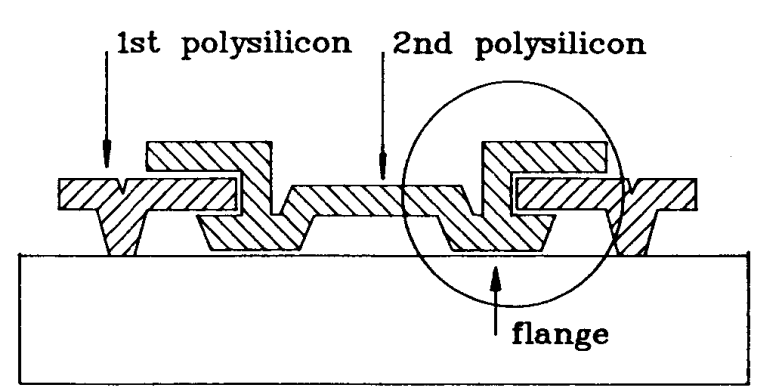

Figure 2 Cross section of the flanged plate slider. The plate can move freely in and out of the paper.

This bridge-slider structure is fabricated using five masks in a sacrificial layer technique. Fabrication starts by coating the four-inch wafers with $1.5-\mu \mathrm{m}$-thick low-pressure-chemicalvapor-deposited (LPCVD) phosphosilicate glass (PSG). Mask 1 is used to open anchor windows for the first-layer polysilicon. The first-layer LPCVD polysilicon is then deposited and patterned using mask 2. Polysilicon is patterned in a $\mathrm{CCl}_{4}$ plasma by reactive-ion-etching (RIE) to provide anisotropic etching. Mask 3 is used to open the flange windows with a timecontrolled PSG etch in 5:1 buffered hydrofloric acid (BHF). This step creates a $1.5 \mu \mathrm{m}$-long undercut of the first-layer-polysilicon restraining element which forms the flanges. A LPCVD siliconnitride sacrificial layer is then deposited to coat all the exposed surfaces including the flange undercuts. This silicon-nitride layer is $200 \mathrm{~nm}$ thick and, therefore, determines the gap between the two polysilicon layers. Mask 4 opens the anchor windows for the bridges. The second-layer polysilicon is then deposited and patterned using mask 5. The last step.which frees the structures in concentrated HF (49\%) takes several hours. For unannealed polysilicon structures, the highest temperature in the fabrication process is $650{ }^{\circ} \mathrm{C}$ which occurs during deposition of the phosphorus-doped polysilicon films. Annealed samples are treated at $1000^{\circ} \mathrm{C}$ for one hour before the final etching.

\section{EXPERIMENTAL PROCEDURE}

Figure 3 demonstrates the fracture experiment. By pushing the slider with a mechanical probe, an initially straight bridge can be buckled until it fractures. Pushing is done under an optical microscope and the whole procedure is video-taped to provide a record. Since the edges of the flanges are shaped with sawtooth scales, the maximum distance $\Delta L$ that the slider can be pushed is easily measured. Figure 4 shows some pre-pushed and buckled bridge-sliders to demonstrate the experiment. The bridges retain their buckled shapes in Fig. 4 because they are jammed in the slider under such high strain. Figure 5 also shows a pre-buckled bridge to demonstrate the clamped boundary conditions used in our theory. Once $\Delta L$ is measured, a nonlinear beam-bending theory is used to interpret the strain in the beam.

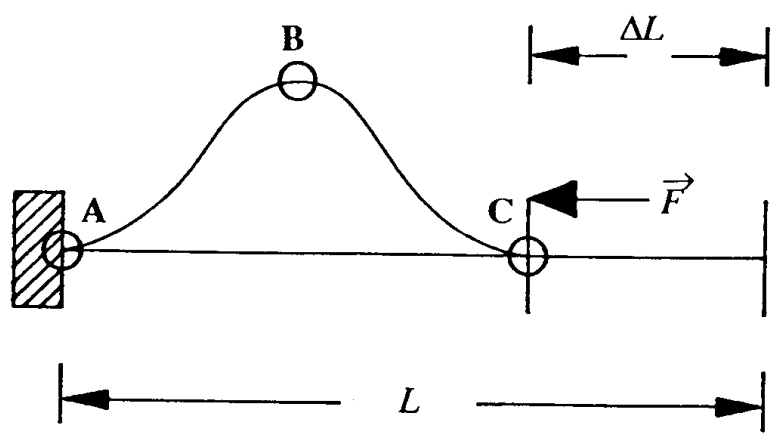

Figure 3 Side view of a buckled beam. The beam is straight before loading. Points $\mathrm{A}, \mathrm{B}$, and $\mathrm{C}$ have the maximum bending moment and therefore are the expected locations of fracture.

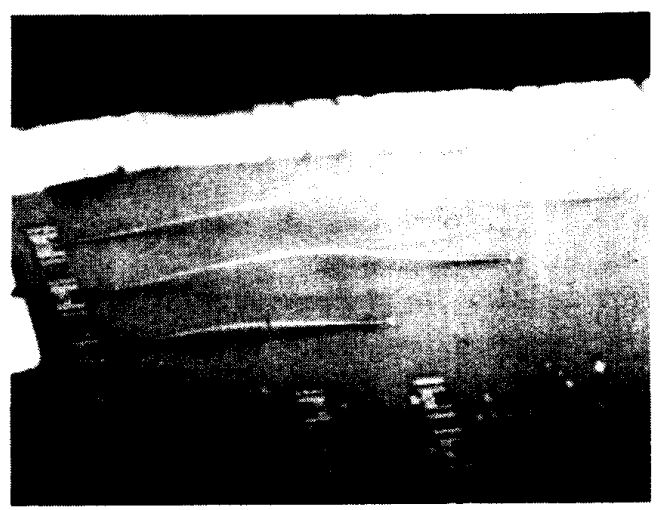

Figure 4 SEM picture of strained bridge-sliders. When sufficiently strained, the bridges retain their buckled shapes. The lengths of the bridges are 4,3 , and 2 $\mathrm{mm}$, respectively, from top to bottom of the picture.

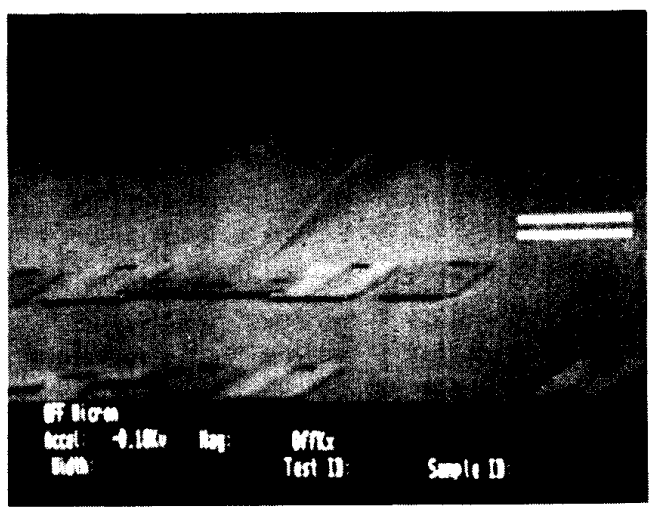

Figure 5 SEM picture of a strained bridge-slider. The buckled shape is a result of clamped boundary conditions. 


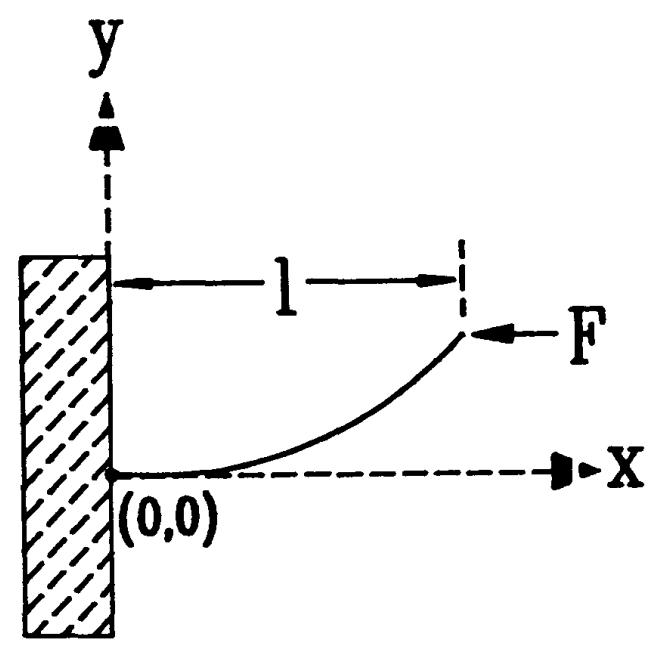

Figure 6 Side view of an axially loaded cantilever.

\section{THEORY}

Because the bridge bending is symmetric (Fig. 3), only one quarter of the structure needs to be analyzed. Figure 6 shows the bending of a cantilever beam due to a horizontal force. The analysis starts with the bending equation [7]

$$
\frac{1}{r(x, y)}=\frac{M(x, y)}{E_{\mathrm{Y}} I}
$$

where the $r(x, y)$ is the curvature, $M(x, y)$ is the bending moment, $E_{\mathrm{Y}}$ is Young's modulus, and $I$ is the moment of inertia. In the case of a rectangular cross section, $I=\frac{w t^{3}}{12}$. Here, $w$ is the beam width and $t$ is the beam thickness. Eq. (1) can be rewritten as

$$
-\frac{y^{\prime \prime}}{\left(1+y^{\prime 2}\right)^{3 / 2}}=\frac{F[y(l)-y]}{E_{\mathrm{Y}} I}
$$

where $l$ is the projection length of the beam on the $x$-axis and $y(l)$ is the beam-end deflection (which will be solved for later). The boundary conditions on Eq. (2) are $y^{\prime}(0)=0$ and $y^{\prime \prime}(l)=0$. The solution for $y(l)$, obtained by solving Eq. (2), is [8]

$$
y(l)=\frac{p L}{2 K(p)}
$$

and intermediate solutions for for $l$ and $F$ are

$$
\begin{gathered}
l=\frac{L-\Delta L}{4}=\frac{L}{4}\left[\frac{2 E(p)}{K(p)}-1\right] \\
F=16 E_{\mathrm{Y}} I \frac{K^{2}(p)}{L^{2}}
\end{gathered}
$$

where $L$ is the total length of the bridge and $p=\frac{y(l)}{2} \sqrt{\frac{F}{E_{\mathrm{Y}} I}}$ is defined to simplify the derivation. The quantities $K(p)$ and $E(p)$ are complete elliptic integrals of the first and second kind, respectively. These two functions are defined by

$$
\begin{gathered}
K(p)=\int_{0}^{\pi / 2}\left(1-p^{2} \sin \phi\right)^{-1 / 2} d \phi \\
E(p)=\int_{0}^{\pi / 2}\left(1-p^{2} \sin \phi\right)^{1 / 2} d \phi
\end{gathered}
$$

For a given value of $p$, both $K(p)$ and $E(p)$ can be obtained from mathematical tables. The parameter $p$ can be obtained using Eq. (4) since $\Delta L$ is measured and $p$ is the only unknown in Eq. (4). Knowing $p$, one can calculate the force $F$ from Eq. (5) and $y(l)$ from Eq. (3).

Once $F$ and $y(l)$ have been calculated, the maximum strain can also be determined. The maximum strain (which occurs at the clamped end) is [7]

$$
\begin{aligned}
\varepsilon_{t \max } & = \pm \frac{M(0,0) t}{2 E_{\mathrm{Y}} I}-\frac{F}{w t E_{\mathrm{Y}}} \\
& = \pm 4 p K(p) \frac{t}{L}-\frac{4}{3} K^{2}(p)\left(\frac{t}{L}\right)^{2}
\end{aligned}
$$

where the + sign stands for tension and the - sign for compression. Since we have used one-dimensional beam theory, strain is independent of beam width $w$. As shown in Fig. 6, the lower half of the polysilicon beam is in tension and the upper part is in compression. In fact, the difference in magnitude between compressive and tensile strains is twice the last term in Eq. (8). This term represents compressive strain caused by horizontal force. It is relatively small compared to the bending strain due to its $\left(\frac{t}{L}\right)^{2}$ dependence. Moreover, in brittle materials, tensile stress is usually regarded as the most significant cause of fracture [6]. Therefore, we consider only the maximum tensile strain which is

$$
\varepsilon_{+\max }=4 p K(p) \frac{t}{L}-\frac{4}{3} K^{2}(p)\left(\frac{t}{L}\right)^{2}
$$

Figure 7 shows the results of theoretical calculations of $\varepsilon_{+\max }$ using Eq. (9) for bridge lengths $L=100,200,300$, and $400 \mu \mathrm{m}$ and thickness $1.27 \mu \mathrm{m}$. Using Fig. 7, one can convert the experimental $\Delta L$ into fracture strain. For example, one finds $\Delta L$ to be $41 \mu \mathrm{m}$ for a bridge that is $200 \mu \mathrm{m}$ long. This value of $\Delta L$ can be used with Fig. 7 to find the corresponding fracture strain which is $1.88 \%$.

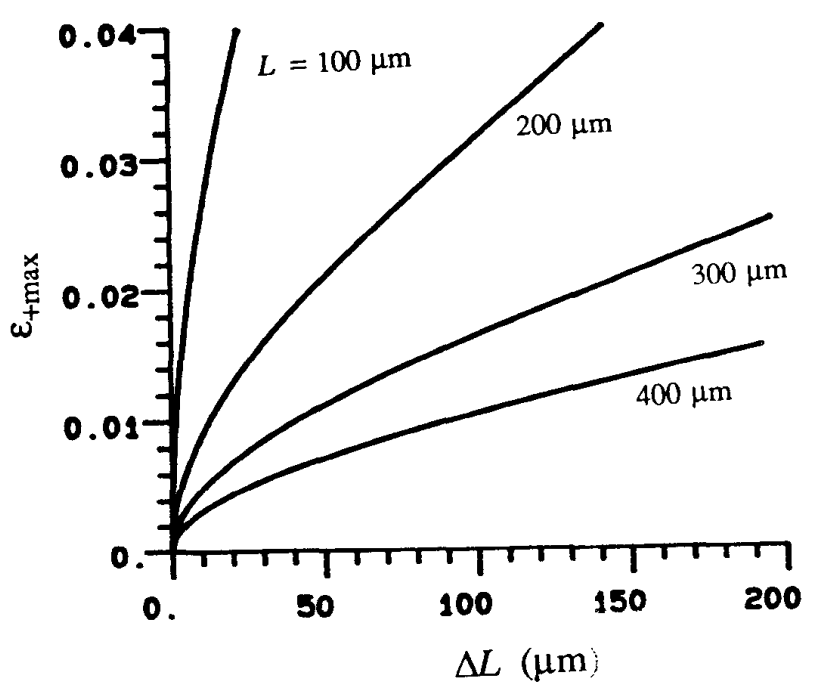

Figure 7 Theoretical calculations of $\varepsilon_{+\max }(\Delta L)$ from Eqs. (4) and (\%) Thickness of the bridges is $1.27 \mu \mathrm{m}$. 
Since the cantilever beam is only one quarter of the full bridge, the maximum strain occurs at the center and the two ends of the bridge as pointed out in Fig. 3. Therefore, the theory predicts that fracture should happen at those three points, as is observed experimentally.

\section{RESULTS}

In brittle materials, fracture occurs when a crack can propagate in the material. Cracks can originate from defects in solids such as impurity inclusions and segregations, and grain boundaries. The probability that these defects will cause fracture depends heavily on their spatial extent, shape, and orientation, which are statistically distributed. Therefore, the study of polysilicon fracture should be done statistically. To facilitate this, we present statistical results obtained from our experiments.

Table 1 lists $\Delta L$ and $\varepsilon_{+\max }$ deduced from the theory presented for both the unannealed and annealed bridge sliders. The highest processing temperature for the unannealed polysilicon is $650^{\circ} \mathrm{C}$. Annealing is carried out at $1000^{\circ} \mathrm{C}$.

For the unannealed bridge-sliders, the average fracture strain is $1.72 \%$ with a $95 \%$ confidence interval bounded by $1.63 \%$ and $1.81 \%$. The variance is $0.217 \%$. For the annealed bridge-sliders, the average fracture strain is $0.93 \%$ and the $95 \%$ confidence interval is $(0.89 \%, 0.97 \%)$ with a variance of $0.1 \%$.

Two important results emerge from this study. One is that the annealed bridge-sliders have a much smaller average fracture strain than do the unannealed samples ( $0.93 \%$ versus $1.72 \%$ ). The second result is that unannealed bridges show larger scatter of the fracture strain in terms of both confidence interval and variance. Our experiments have shown that high-temperature annealing has, therefore, decreased both the magnitude and distribution of the fracture strain of polysilicon.

\section{REFERENCE}

[1] L. S. Fan, Y. C. Tai and R. S. Muller, "Pin joints, gears, springs, cranks, and other novel micromechanical structures," Transducers' 87 , 4th Intl. Conf. on Solid-State Sensors and Actuators, Tokyo, Japan (June 3-5, 1987), pp.

849-852.

[2] K. J. Gabriel, W. S. N. Trimmer, and M. Mehregany, "Micro gears and turbines etched from silicon," Transducers' 87, 4th Intl. Conf. on Solid-State Sensors and Actuators, Tokyo, Japan (June 3-5, 1987), pp. 853-856.

[3] R. L. Eisner, "Tensile tests on silicon whiskers," Acta Metallurgica, Vol. 3, 1955, pp.414-415.

[4] G. L. Pearson, W. T. Read Jr, and W. L. Feldmann, "Deformation and fracture of small silicon crystals," Acta Metallurgica, Vol. 5, April, 1955, pp.181-191.

[5] L. S. Fan, Y. C. Tai, and R. S. Muller, "Integrated movable micromechanical structures for sensors and actuators," IEEE Trans. ED, (to appear June, 1988).

[6] A. de S. Jayatilaka, "Fracture of engineering brittle materials," London, Applied Science Publishers LTD, 1979.

[7] S. Timoshenko and D. H. Young, "Elements of strength of materials," Princeton, N.J., D. Van Nostrand Co., 1968.

[8] R. Frisch-Fay, "Flexible bars," Washington, Butterworth Inc., 1962.

Table 1 Experimental fracture strain of polysilicon

\begin{tabular}{|c|l|l|l|l|}
\hline \multicolumn{1}{|c|}{ Size } & \multicolumn{3}{|c|}{ Unannealed } & \multicolumn{2}{c|}{ Annealed } \\
\hline \multicolumn{1}{|c|}{$L / w / t$} & \multicolumn{1}{|c|}{$\Delta L(\mu \mathrm{m})$} & \multicolumn{1}{|c|}{$\varepsilon_{+\max }(\%)$} & \multicolumn{1}{c|}{$\Delta(\mu \mathrm{m})$} & $\varepsilon_{+\max }(\%)$ \\
\hline $100 / 2 / 1.27$ & 5,7 & $1.80,2.14$ & $\mathrm{x}$ & $\mathrm{x}$ \\
$100 / 6 / 1.27$ & 8 & 2.29 & $\mathrm{x}$ & $\mathrm{x}$ \\
$100 / 10 / 1.27$ & $\mathrm{x}$ & $\mathrm{x}$ & $\mathrm{x}$ & $\mathrm{x}$ \\
$100 / 20 / 1.27$ & $\mathrm{x}$ & $\mathrm{x}$ & $\mathrm{x}$ & $\mathrm{x}$ \\
\hline $200 / 2 / 1.27$ & 41 & 1.88 & 10,16 & $0.90,1.14$ \\
$200 / 6 / 1.27$ & 36,40 & $1.75,1.86$ & 11,15 & $0.94,1.11$ \\
$200 / 10 / 1.27$ & $27,29,33,37$ & $1.51,1.56,1.67,1.78$ & 10,11 & $0.90,0.94$ \\
$200 / 20 / 1.27$ & $29,31,35,37,41$ & $1.56,1.62,1.73,1.78,1.88$ & 9,10 & $0.85,0.90$ \\
\hline $300 / 2 / 1.27$ & 124 & 1.87 & 35,36 & $0.93,0.94$ \\
$300 / 6 / 1.27$ & 75,100 & $1.40,1.65$ & 30,45 & $0.86,1.06$ \\
$300 / 10 / 1.27$ & $77,95,97$ & $1.42,1.60,1.62$ & 38,38 & $0.97,0.97$ \\
$300 / 20 / 1.27$ & 85 & 1.50 & 23,27 & $0.75,0.81$ \\
\hline $400 / 2 / 1.27$ & $\mathrm{x}$ & $\mathrm{x}$ & 90,94 & $0.99,1.01$ \\
$400 / 6 / 1.27$ & $\mathrm{x}$ & $\mathrm{x}$ & 74,84 & $0.89,0.95$ \\
$400 / 10 / 1.27$ & $\mathrm{x}$ & $\mathrm{x}$ & 79,84 & $0.92,0.95$ \\
$400 / 20 / 1.27$ & $\mathrm{x}$ & $\mathrm{x}$ & 53,60 & $0.75,0.80$ \\
\hline
\end{tabular}

